OAl-PMH: http://www.indteca.com/ojs/index.php/Revista Scientific/oai

Artículo Original / Original Article

\title{
Estilos de Apego en Mujeres Víctimas de Violencia
}

Autoras: Carmen Virginia Polit Cadena Universidad Internacional SEK, UISEK cvpolit.mpp@uisek.edu.ec Quito, Ecuador https://orcid.org/0000-0003-4903-6576

Rita Alexandra Proaño Echeverría Universidad Internacional SEK, UISEK raproano.mpp@uisek.edu.ec Quito, Ecuador https://orcid.org/0000-0001-5297-7057

Resumen

La violencia contra la mujer ha sido tipificada como un grave problema de salud pública con elevados índices que varían de acuerdo con la región. Existen muchos factores asociados, tales como el abuso de alcohol, depresión, patrones familiares de violencia de género que representan experiencias vividas de violencia, entre estos, el apego a la pareja es uno de los más frecuentes y se constituye una barrera para no afrontar una relación cargada de maltrato y violencia. Esta investigación la cual tiene un enfoque cualitativo y de tipo descriptivo, y cuyo objetivo es describir el estilo de apego predominante en mujeres víctimas de violencia a través de la aplicación de pruebas psicométricas, para contribuir a mejorar el abordaje terapéutico. Se realizó un estudio de campo, con 28 mujeres víctimas de violencia por parte de su pareja que acudieron a la Fundación Nosotras con Equidad en la ciudad Riobamba, Ecuador. Los datos se obtuvieron a través de la aplicación del Cuestionario de Evaluación del apego adulto de Melero y Cantero (2008), se analizaron con frecuencias absolutas y relativas. A través de los resultados, se pudo determinar el estilo de apego entre las mujeres participantes, siendo el más predominante el estilo huidizo-temeroso, seguido de huidizo-alejado y preocupado. El estilo afectivo-seguro se presentó en la escala muy bajo.

Palabras clave: violencia doméstica; mujeres; derechos humanos. Código de clasificación internacional: 6105.05 - Psicometría.

Cómo citar este artículo:

Polit, C., \& Proaño, R. (2021). Estilos de Apego en Mujeres Víctimas de Violencia. Revista Scientific, 6(22), 21-37, e-ISSN: 2542-2987. Recuperado de: https://doi.org/10.29394/Scientific.issn.2542-2987.2021.6.22.1.21-37

Fecha de Recepción: 05-01-2021
Fecha de Aceptación: 07-06-2021
Fecha de Publicación: 05-11-2021 
OAl-PMH: http://www.indteca.com/ojs/index.php/Revista Scientific/oai

Artículo Original / Original Article

Attachment Styles in Women Victims of Violence

\begin{abstract}
Violence against women has been classified as a serious public health problem with high rates that vary according to the region. There are many associated factors, such as alcohol abuse, depression, family patterns of gender violence that represent lived experiences of violence, among these, attachment to the partner is one of the most frequent and constitutes a barrier to not face a relationship fraught with abuse and violence. This research which has a qualitative and descriptive approach, and whose objective is to describe the predominant attachment style in women victims of violence through the application of psychometric tests, to help improve the therapeutic approach. A field study was carried out with 28 women victims of violence by their partner who attended the Nosotras con Equidad Foundation in the city of Riobamba, Ecuador. The data were obtained through the application of the Adult Attachment Assessment Questionnaire by Melero and Cantero (2008), they were analyzed with absolute and relative frequencies. Through the results, it was possible to determine the attachment style among the participating women, the most predominant being the elusive-fearful style, followed by elusive-withdrawn and worried. The affective-safe style was presented on the very low scale.
\end{abstract}

Keywords: domestic violence; women; human rights. International classification code: 6105.05 - Psychometrics.

\footnotetext{
How to cite this article:

Polit, C., \& Proaño, R. (2021). Attachment Styles in Women Victims of Violence. Revista Scientific, 6(22), 21-37, e-ISSN: 2542-2987. Recovered from: https://doi.org/10.29394/Scientific.issn.2542$\underline{2987.2021 .6 .22 .1 .21-37}$
}

Date Received: 05-01-2021
Date Acceptance: 07-06-2021
Date Publication: 05-11-2021 


\section{Introducción}

La violencia contra las mujeres, especialmente en las relaciones de pareja, es un problema grave que se encuentra en aumento en nuestra sociedad. Es posible que muchos no se denuncien, por lo tanto, las cifras pueden presentar una subestimación importante. Para optimizar el abordaje del problema de la violencia hacia la mujer, es imprescindible conocer sus dimensiones, los factores asociados por los cuales las mujeres permanecen 0 regresan constantemente con sus parejas, aunque reciben nuevamente acciones de violencia de cualquier índole; se trata de relaciones tóxicas, sin poder superar los obstáculos que les limitan para alejarse de este tipo de relación.

Según la Organización Mundial de la Salud (OMS, 2013): la violencia contra la mujer se ha convertido en un grave problema de salud pública a nivel mundial, principalmente porque se traduce en una violación a los derechos humanos fundamentales, los cuales incluyen derecho a la salud, educación, participación en cuestiones de política, disfrutar de bienestar económico y el no ser objeto de violencia, entre otros.

Esta organización ha estimado las cifras de mujeres que han sido víctimas de violencia, las cuales ascienden a $35 \%$ a nivel mundial, tanto por su pareja como por otra persona, siendo el primero el más frecuente. Un dato importante son las cifras de homicidios producto de la violencia conyugal, la cual se ha calculado en el $38 \%$.

En un estudio realizado por Guedes, García-Moreno y Bott (2014): que abarcó 12 países de Latinoamérica y el Caribe, determinaron diferentes aspectos de la violencia contra la mujer, resultando una situación similar a la que se maneja a nivel mundial, con cifras globales del $36 \%$ de este problema, esta puede variar en algunos países, por ejemplo, en Bolivia se maneja el $25,5 \%$, República Dominicana maneja cifras de 17\%, Jamaica 7,7\%. Las mujeres con secuelas de lesiones por violencia conyugal se registran en $41,2 \%$ 
en Honduras y $81,6 \%$ en Paraguay.

La ideación suicida en mujeres víctimas de violencia, se estimó en Colombia en $23 \%$ y El Salvador en 31,7\%. Adicionalmente, el porcentaje de mujeres que aceptan o justifican que el esposo golpee a la esposa en circunstancias específicas asciende al $38,2 \%$ en Ecuador y 2,9\% en Jamaica.

En Ecuador, como Indica el Consejo Nacional para la Igualdad de Género (CNIG, 2014): la violencia contra la mujer se ha transformado en un problema social muy grave, estimando que seis de cada 10 mujeres mayores de 15 años han sido víctima de agresiones de índole físicas, psicológicas, sexuales o patrimoniales, siendo la forma más frecuente la psicológica con registros que alcanzan hasta el 53,9\%; en segundo lugar la violencia física con el $38 \%$; en tercer lugar por la violencia sexual con $25,7 \%$ y la violencia patrimonial con el $16,7 \%$.

La teoría del apego, señalando a Bowlby (1998): ha sido el puntal para poder iniciar estudios sobre esta problemática que viene carcomiendo a nuestra sociedad la cual es definida como la propensión presente en los seres humanos, para desarrollar vínculos afectivos con otras personas, lo cual permite conocer algunas formas de trastornos emocionales y explicar ciertas alteraciones de personalidad, como la ansiedad, la ira, la depresión y el desapego emocional.

Continúa afirmando este autor que la experiencia que vive el niño en sus primeros años es fundamental y genera un efecto que puede permanecer a largo plazo, siendo positivo para su salud mental si esta relación inicial es íntima y cálida, sin embargo, si existe una relación de maltrato tendrá consecuencias negativas en el niño, desarrollando un patrón de apego que depende del vínculo que presenta con su madre. Para Lucariello (2012), es posible clasificar los tipos de apego en:

- Apego seguro: presente en personas que mantienen relaciones afectivas alegres, cargadas de emociones positivas, tienen a confiar en 
OAI-PMH: http://www.indteca.com/ojs/index.php/Revista Scientific/oai

Artículo Original / Original Article

su pareja y la aceptan con todos sus defectos, generan capacidad para la resolución de conflictos interpersonales

- Apego preocupado: en estos casos hay mayor tendencia a sentir cólera, tristeza, sensaciones de miedo, vergüenza, ansiedad, angustia, indefensión, sobrexcitación, utilizan el miedo o la rabia como una estrategia para reclamar la cercanía, mecanismo aprendido en sus relaciones familiares durante la infancia

- Apego rechazante: se relaciona con emociones negativas que involucran desprecio, tristeza, dificultad para la alegría y les es difícil reconocer emociones y sentimientos propios, se muestran inseguros acerca de lo que sienten hacia la figura de apego y no son conscientes de su propia hostilidad, adicionalmente, suelen negar sus necesidades afectivas usando distractores como el trabajo y el alcohol para no focalizar su atención en los sentimientos

- Apego temeroso: presentan signos de dificultad para superar experiencias traumáticas ante la pérdida de algún ser involucrado en una relación de apego, en su infancia, las relaciones de apego presentaron desamparo, disforia y coerción.

Interpretando a Finkel y Slotter (2007): en algunos casos, el apego permite la existencia de altos niveles de interdependencia emocional y conductual que caracterizan a un grupo de parejas, las cuales presentan niveles inusualmente elevados de conflictos que a pesar de no ser inicialmente violentos, en ocasiones, pueden servir como precursor del comportamiento violento.

La violencia es en este caso, un comportamiento impulsivo que emerge cuando los individuos, tanto hombres como mujeres, se sienten enojados o amenazados en su vínculo de apego, principalmente si uno de ellos se siente 
ansiosamente unido al otro. Esa actitud es más común en quienes desarrollaron un gran apego por su cuidador durante la infancia.

Para Levendosky, Lannert y Yalch (2012): existe el riesgo de que la violencia entre las parejas sea transmitida entre generaciones a los niños expuestos, y puede replicar la actitud como víctimas o perpetradores, estos niños presentan resultados desadaptativos tras experiencias de violencia hacia su madre, desarrollando conductas de apegos.

En el estudio de Lucariello y Fajardo (2012): los estilos de amor en mujeres maltratadas, se refiere a que "la teoría del apego puede ser de gran utilidad a la hora de explicar la manutención del círculo del maltrato y las dificultades que presentan las mujeres en salir de esta relación nociva" (pág. 348); estos incluyen la ansiedad por una separación y el consecuente temor que genera la pérdida, elementos que logran impedir que la víctima pueda evaluar con objetividad las ventajas y desventajas de su situación, por lo que las gobierna la incertidumbre sobre el futuro y se cuestionan la posibilidad de poder superarse.

Al estudiar estos procesos es posible definir dos tipos de apego: apego ansiedad que se caracteriza por una preocupación, miedo al rechazo y abandono de la pareja, el apego evitación que genera preocupación con el grado en que una persona se llegue a sentir siendo dependiente de otra.

Otro estudio realizado en Chile por Loubat, Ponce y Salas (2007): indagó el estilo de apego que presentaban un grupo de mujeres víctimas de violencia conyugal y la influencia de éste para mantenerse en la relación, participaron 50 mujeres en un estudio correlacional comparativo, el diseño fue cuasi experimental y se les administró el cuestionario CaMir. Los resultados exponen que las mujeres con violencia conyugal presentan estilo de apego preocupado y las características de este apego influyen en la capacidad de afrontamiento hacia la violencia y por consecuencia se mantienen en el maltrato. 
La teoría del apego ha sido clave y es por eso que otro estudio de Zebardast y Mahmoud (2013): comparó los estilos de apego y las dimensiones de la personalidad en mujeres que son víctimas de violencia familiar, el estudio fue causal-comparativo, incluyó a todas las mujeres que acudían al tribunal de familia de la localidad como víctimas de violencia durante el año 2010. La prueba estadística descriptiva fue el chi cuadrado y análisis multivariado de varianza.

Los resultados demostraron una diferencia significativa entre las mujeres víctimas de violencia en todas las dimensiones de la personalidad. En conclusión, el estilo de apego inseguro en mujeres que sufrieron violencia no solo causó una relación fría, sino que también redujeron la devoción y el nivel de compromiso, presentaron altos niveles de neurosis, bajo nivel de amabilidad, falta de flexibilidad y niveles bajos de extravagancia.

En el estudio de Vega (2015): donde se utilizó el cuestionario de Apego Adulto, fue aplicado a una muestra de 197 personas, los resultados obtenidos muestran que el estilo de apego predominante estuvo caracterizado por la resolución hostil de conflicto, rencor y posesividad, los participantes no se identifican con un estilo afectivo exclusivo, sino que mantienen características de los todos estilos.

Este estudio permite revisar el uso del Cuestionario de Apego Adulto establecido para este trabajo de investigación, con lo cual es posible medir los resultados que han obtenidos otros investigadores, los cuales servirán de aporte para la discusión de estos.

En base a lo anterior expuesto, podemos decir que si el problema de la mujer víctima de violencia no es resuelto, las consecuencias no solo son negativas para el individuo, en el cual las áreas afectadas pueden ser física, emocional y psicológica, las relaciones interpersonales y el desarrollo como persona; adicionalmente, la familia y la sociedad igualmente se afectan como consecuencia de replicar en los hijos las relaciones de violencia, generando 
nuevamente más individuos violentos o víctimas del mismo.

En Ecuador se han realizado diversos estudios acerca de la violencia contra la mujer, sin embargo, algunos aspectos tales como los estilos de apego y su relación con la violencia en la pareja requieren ser profundizados, por lo tanto, la evaluación del estilo de apego en las relaciones de violencia contra la mujer permitirá obtener datos relevantes, mejorando la caracterización y el conocimiento que se tiene hasta la fecha sobre esta situación.

Si no se evalúa el estilo de apego que presentan las mujeres víctimas de violencia, se estaría dejando un vacío de información importante, tomando en cuenta que el estilo de apego puede representar un factor importante en el afrontamiento de una relación violenta y por lo tanto, constituirse como un limitante para buscar ayuda, el estudio de estos aspectos servirá para optimizar la comprensión de esta problemática y puede aportar elementos para plantear nuevas y mejores estrategias de evaluación y tratamiento.

La violencia contra las mujeres, especialmente en las relaciones de pareja, es un flagelo que aumenta en nuestra sociedad y las acciones al respecto aún no han logrado disminuir la cantidad de víctimas existentes. Muchos casos es posible que no se denuncien, por lo tanto, las cifras pueden presentar una subestimación importante.

El presente estudio tiene la finalidad de evaluar los estilos de apego y establecer el o los estilos predominantes en mujeres víctimas de violencia aplicando pruebas psicométricas para contribuir a mejorar el abordaje terapéutico.

Para mejorar el abordaje del problema de la violencia hacia la mujer, es imprescindible conocer sus dimensiones, los factores asociados por los cuales las mujeres permanecen o regresan constantemente, aunque vuelvan a recibir acciones de violencia de cualquier índole; se trata de relaciones toxicas, sin poder superar las barreas que les limitan para alejarse de este tipo de relación.

Es importante conocer los estilos de apego predominantes que limitan 
a la mujer para afrontar una relación de violencia y maltrato, considerando la teoría del apego como un soporte para comprender la violencia contra la mujer.

\section{Metodología}

La presente investigación se realizó mediante un enfoque cualitativo, de tipo descriptivo, con la cual se indagaron detalles del problema estudiando, los elementos que lo constituyen y sus características. Se trata de un estudio transversal por lo que se obtuvieron los datos en un momento específico y actual.

La modalidad del presente trabajo de investigación fue de campo o in situ, en virtud de que los datos fueron recogidos directamente del sitio donde se encuentra el objeto de estudio, es decir, en la institución donde acuden para formular la denuncia respectiva.

El análisis se realizó en una población de 28 mujeres víctimas de violencia que acuden a una Fundación Nosotras con Equidad en la ciudad de Riobamba, seleccionadas por conveniencia

La medición se realizó a través de la aplicación del Cuestionario de Apego Adulto de Melero y Cantero (2008): cuestionario que busca identificar los cuatro estilos principales de apego, cuyas características principales incluyen los estilos: afectivo-seguro, huidizo-alejado, preocupado y huidizotemeroso. El cuestionario fue aplicado a un total de 445 personas en España, para garantizar la representatividad de la muestra. Está formada por 20 ítems con una escala de respuesta tipo Likert de 6 puntos: uno completamente en desacuerdo hasta seis completamente de acuerdo.

\section{Resultados}

El grupo etario más frecuente fue el de mayores de 41 años con el $39,3 \%$ de las participantes, seguido de 35 a 40 años donde se agrupó el 32,1\% y de 26 a 30 años el 14,3\% de la muestra. Los estilos de apego evidenciados 
en la muestra de mujeres participantes en la investigación fueron:

Preocupado: este estilo de apego se presentó muy alto y alto en el $3,6 \%$, cada uno, moderado/alto en el $28,6 \%$ y moderado en el $10,7 \%$, lo cual sumado agrupan el $46,5 \%$ del total de la muestra, es decir, aproximadamente la mitad de las mujeres víctimas de violencia presentan un estilo de apego preocupado, es decir, se trata de un apego que se caracteriza porque utilizan el miedo y la rabia para reclamar la cercanía, siendo estos mecanismos aprendidos durante la infancia. Mientras que el 53,6\% restante registra este estilo de apego entre bajo/moderado a muy bajo, como se puede ver en el gráfico 1.

Gráfico 1. Frecuencia absoluta del estilo de apego Preocupado.

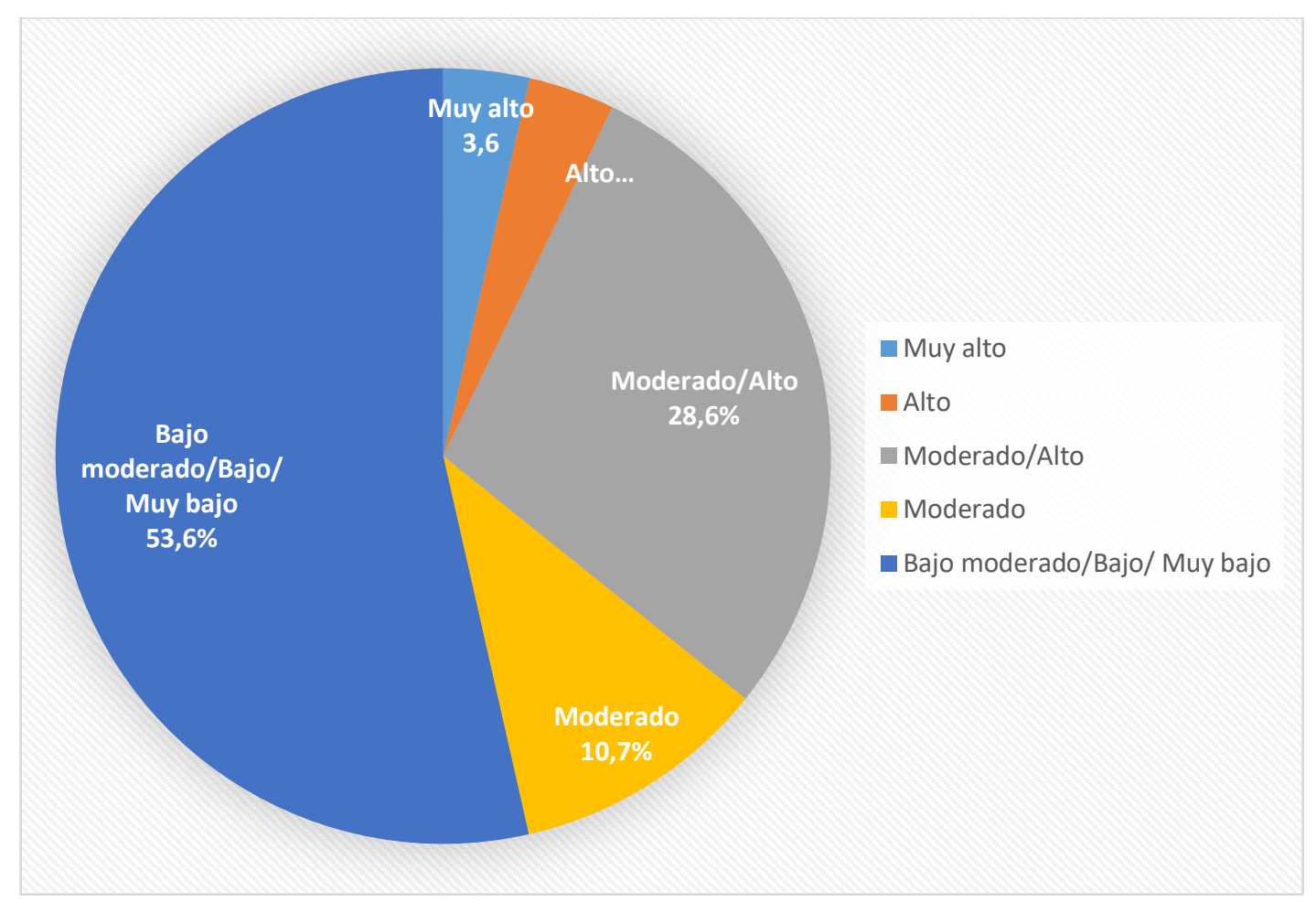

Fuente: Las Autoras (2019).

Huidizo-temeroso: se presentó muy alto en el $17,9 \%$ de las mujeres, $3,6 \%$ en alto, el $25 \%$ para moderado y moderado alto, al agrupar estos datos 
se obtiene que el $71,5 \%$ de las participantes presentan un estilo de apego huidizo-temeroso, según las características de este estilo de apego, son mujeres que tienen dificultad para superar experiencias traumáticas en una relación de apego. Respecto al 28,5\% restantes se distribuyen entre un apego huidizo-temeroso bajo moderado a muy bajo, expuesto en el gráfico 2.

Gráfico 2. Frecuencia absoluta del estilo de apego Huidizo-Temeroso.

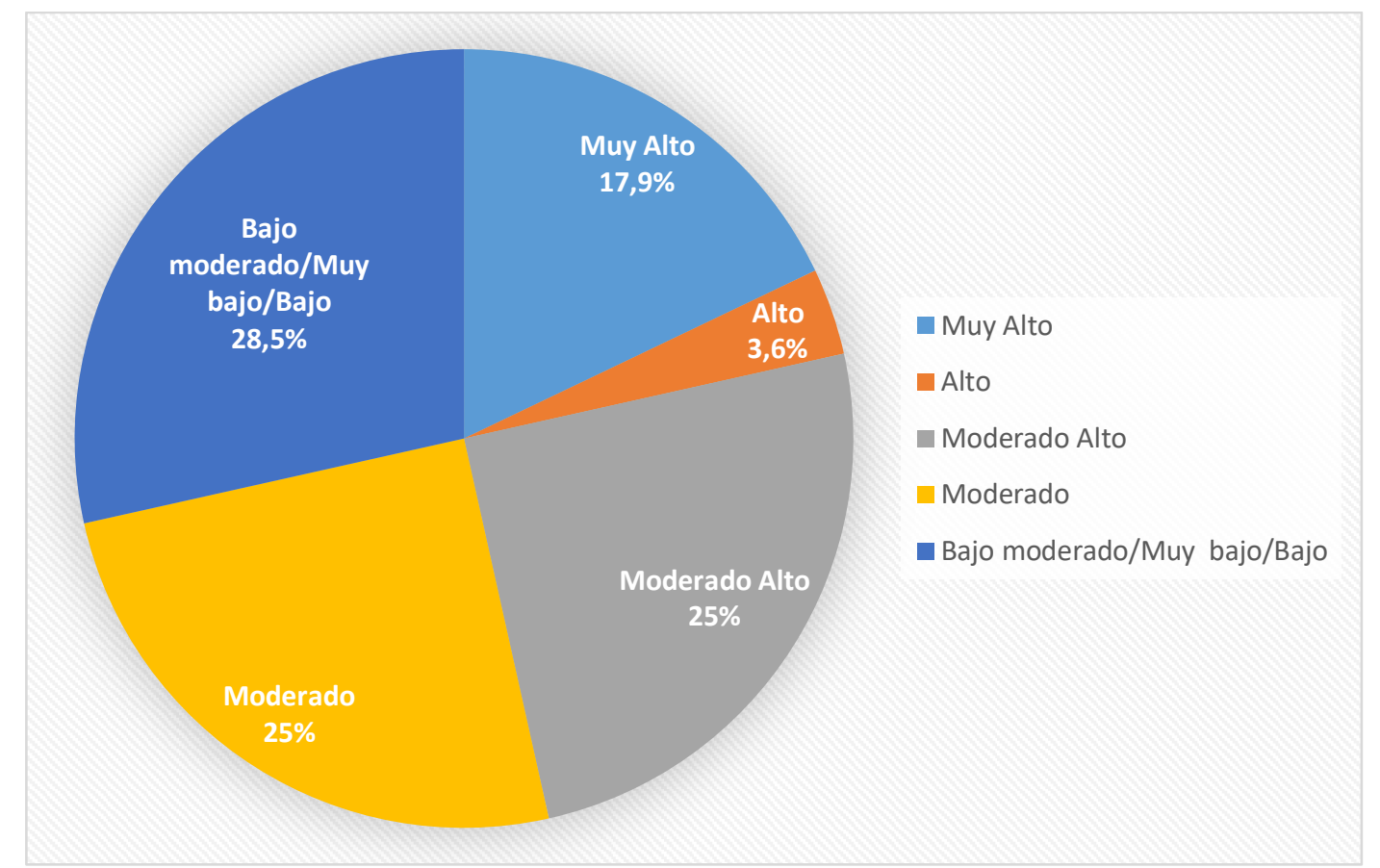

Fuente: Las Autoras (2019).

Afectivo-seguro: este estilo de apegó se registró en todas las participantes del estudio en la categoría muy bajo con el $100 \%$ en la escala del cuestionario, lo cual indica que el total de las mujeres víctimas de violencia no presentan de manera significativa este tipo de apego, es decir, no tienen relaciones afectivas con emociones positivas, desconfían de sus parejas y con dificultad en la resolución de conflictos, como se presenta en el gráfico 3. 
OAI-PMH: http://www.indteca.com/ojs/index.php/Revista Scientific/oai

Artículo Original / Original Article

Gráfico 3. Frecuencia absoluta del estilo de apego Afectivo-Seguro.

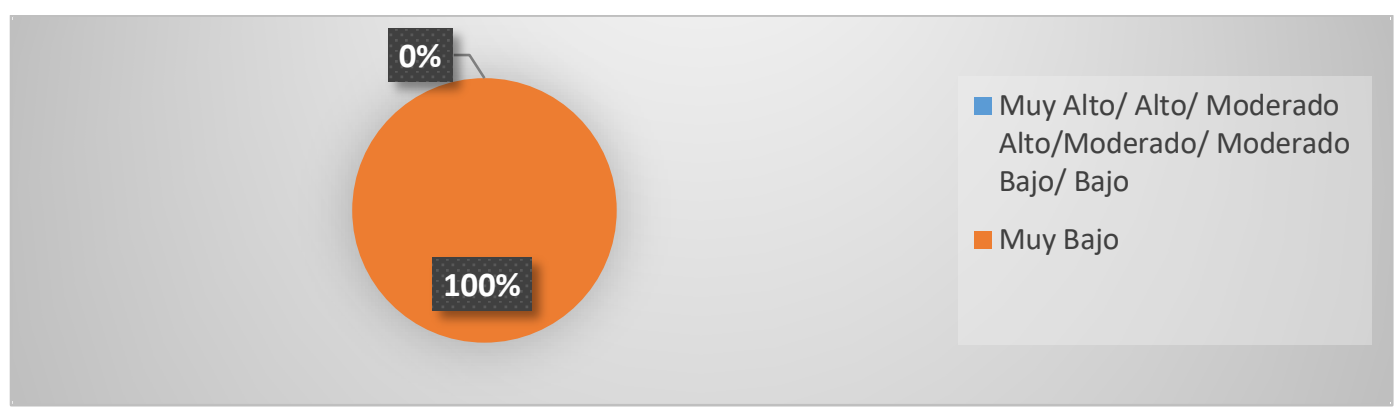

Fuente: Las Autoras (2019).

Huidizo-alejado: se registró muy alto en el 7,1\%, alto y moderado alto en el $14,3 \%$ respectivamente y moderado en el $17,9 \%$. Entre muy alto a Bajo, Muy bajo y moderado, se registra un total del $53,6 \%$ de la muestra. Este estilo de apego tiene como elementos principales emociones negativas que involucran desprecio, inseguridad, negando sus necesidades afectivas a través de distractores para no focalizar su atención en los sentimientos. El $46,5 \%$ restante presenta este estilo de apego distribuido desde bajo/moderado a muy bajo, demostrado en el gráfico 4 .

Gráfico 4. Frecuencia absoluta del estilo de apego Huidizo-Alejado.

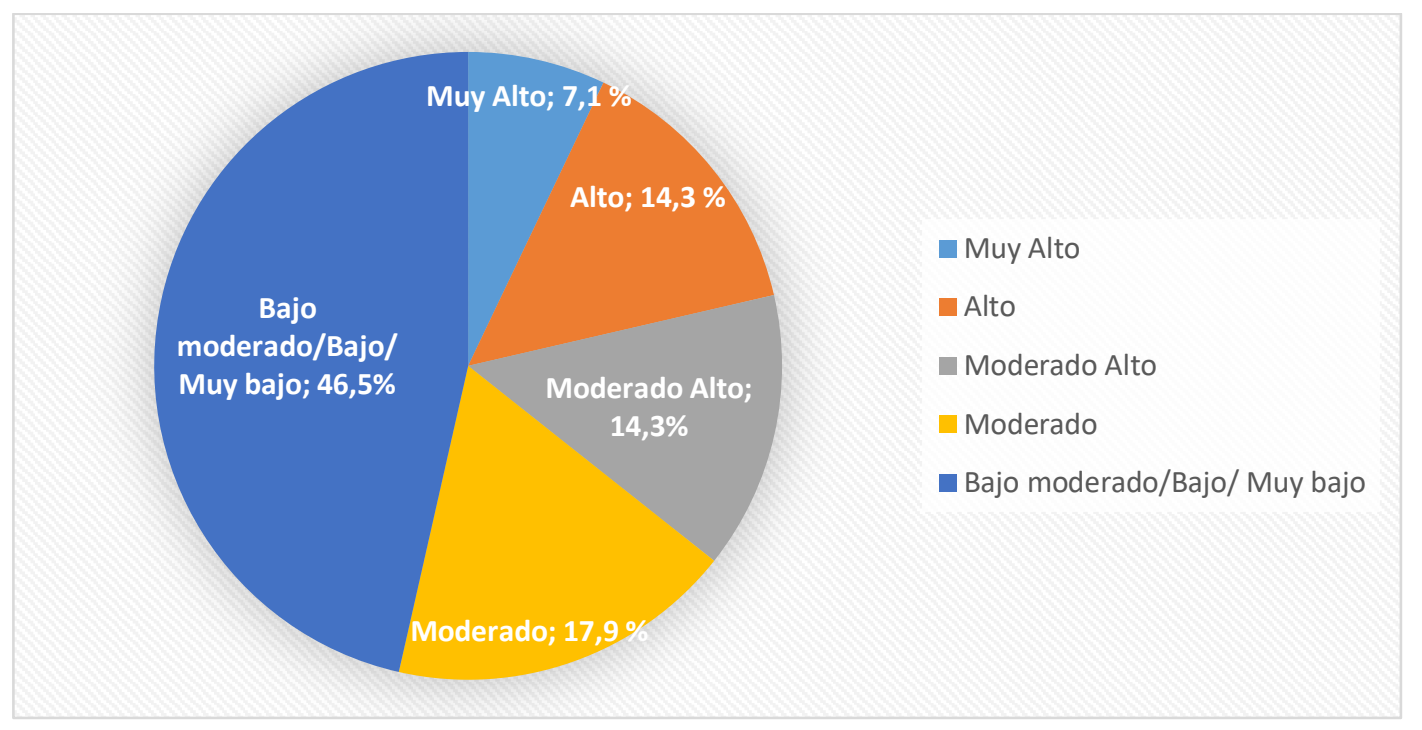

Fuente: Las Autoras (2019). 


\section{Conclusiones}

La presente investigación demuestra que los diferentes estilos de apegos son un eje fundamental para conocer la permanencia de las mujeres en una relación y pueden presentarse de manera conjunta en una persona, manifestándose en niveles diferentes con predominio o no de uno en especial.

Se logra identificar los cuatro estilos de apego, siendo los predominantes aquellos que se caracterizan por haber tenido relaciones iniciales de crianza y cuidado muy deficientes y poco preocupados y dan a notar que las consecuencias de presenciar acciones violentas desde la infancia, puede ser replicado en la vida de pareja. Otro punto importante es que la experiencia que vive el niño en sus primeros años es fundamental, siendo positivo para su salud mental si esta relación inicial es íntima y cálida, por el contrario, si existe una relación de maltrato tendrá consecuencias negativas en el niño, desarrollando un patrón de apego que depende del vínculo que presenta con su madre.

En el caso de las mujeres víctimas de violencia, el apego puede convertirse en una herramienta clave, para mejorar las estrategias de prevención, y abordaje de este problema, al establecer la presencia de estilos de apegos en las relaciones de pareja con violencia.

Este trabajo tiene relevancia social, en virtud de que, con base a los resultados se pueden generar mecanismos de intervención psicoterapéutica para estas pacientes, para identificar su estilo de apego predominante y trabajar en programas que promuevan y permitan a la mujer que es víctima de una relación violenta, afianzar la confianza y tener una autoestima adecuada que le pueda ayudar a salir de este círculo en el que vive.

Finalmente se recomienda realizar estudios más extensos y profundos que abarquen una muestra más grande de participantes, así como la realización en diferentes centros para poder obtener datos que pudieran generalizarse, y de esta manera, caracterizar el problema de la violencia 
contra la mujer y su relación con los estilos de apegos.

Además, utilizar las evaluaciones de los estilos de apego como riesgo para predecir la propensión a ser víctima de una relación violenta, estableciendo la presencia de apego como un posible factor de riesgo.

En el abordaje de las mujeres víctimas de violencia de sus parejas, tomar en cuenta como factores importantes, las relaciones con la madre en la infancia como base fundamental del desarrollo de los estilos de apego en la edad adulta, así como el antecedente de presenciar relaciones de violencia en las familias de origen.

\section{Referencias}

Bowlby, J. (1998). El apego y la pérdida. 1ra edición, ISBN: 9788449306006. Barcelona, España: Paidós Ibérica, S.A.

CNIG (2014). Violencia de género contra las mujeres en el Ecuador: Análisis de los resultados de la Encuesta Nacional sobre Relaciones Familiares y Violencia de Género contra las Mujeres. ISBN: 978-9942-07-761-5. Quito, Ecuador: Consejo Nacional para la Igualdad de Género.

Finkel, E., \& Slotter, E. (2007). An Attachment Theory Perspective on the Perpetuation of Intimate Partner Violence. DePaul Law Review, 56(3), 895-907, e-ISSN: 0011-7188. Recovered from:

https://via.library.depaul.edu/law-review/vol56/iss3/7/

Guedes, A., García-Moreno, C., \& Bott, S. (2014). Violencia contra las mujeres en Latinoamérica y el Caribe. Foreign Affairs Latinoamérica, 14(1), 41-48, ISSN: 1665-1707. México: Instituto Tecnológico Autónomo de México.

Levendosky, A., Lannert, B., \& Yalch, M. (2012). The Effects of Intimate Partner Violence on Women and Child Survivors: An Attachment Perspective. Psychodynamic Psychiatry, 40(3), 397-433, e-ISSN: 
2162-2590. Recovered from:

https://doi.org/10.1521/pdps.2012.40.3.397

Loubat, M., Ponce, P., \& Salas, P. (2007). Estilo de Apego en Mujeres y su

Relación con el Fenómeno del Maltrato Conyugal. Terapia

Psicológica, 25(2), 113-121, e-ISSN: 0716-6184. Recuperado de:

https://www.redalyc.org/articulo.oa?id=78525202

Lucariello, E. (2012). Los tipos de amor y las dimensiones de apego en las

mujeres víctimas del maltrato. Tesis doctoral. España: Universidad

de Extremadura. Recuperado de: http://hdl.handle.net/10662/367

Lucariello, E., \& Fajardo, M. (2012). Estilos de amor en mujeres

maltratadas. International Journal of Developmental and Educational

Psychology, 2(1), 345-360, e-ISSN: 0214-9877. Recuperado de:

https://www.redalyc.org/articulo.oa?id=349832339036

Melero, R., \& Cantero, M. (2008). Los estilos afectivos en la población española: un cuestionario de evaluación del apego adulto. Clínica y salud, 19(1), 83-100, e-ISSN: 2174-0550. España: Colegio Oficial de Psicólogos de Madrid.

OMS (2013). Estimaciones mundiales y regionales de la violencia contra la mujer: prevalencia y efectos de la violencia conyugal y de la violencia sexual no conyugal en la salud: resumen de orientación. WHO/RHR/HRP/13.06. Suiza: Organización Mundial de la Salud.

Vega, R. (2015). Apego, relaciones de pareja y salud psicológica de militares desplegados en Líbano. Tesis Doctoral. Sevilla, España: Universidad de Sevilla.

Zebardast, M., \& Mahmoud, M. (2013). Comparison of attachment styles and personality Sides between women who are victim of domestic violence and ordinary women. Procedia. Social and Behavioral Sciences, 84(9), 1005-1009, e-ISSN: 1877-0428. Recovered from: https://doi.org/10.1016/j.sbspro.2013.06.689 


\section{Carmen Virginia Polit Cadena}

e-mail: cvpolit.mpp@uisek.edu.ec

Nacida en Portoviejo, Ecuador, el 25 de febrero del año

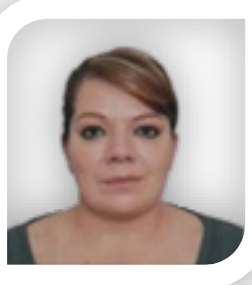
1974. Estudié en la Universidad Técnica Particular de Loja (UTPL), obtuve el título de Licenciada en Psicología General; mis estudios de cuarto nivel los realicé en la Universidad Internacional SEK (UISEK), obteniendo el título de Magister en Psicología con mención en Psicoterapia; ejerzo en consulta privada con niños, adolescentes y adultos; soy Psicóloga voluntaria en el Centro de Atención Psicológica (CAP) de la Universidad Internacional SEK, desde el año 2019. 
Rita Alexandra Proaño Echeverría

email: raproano.mpp@uisek.edu.ec

Nacida en Quito, Ecuador, el 15 de mayo del año 1965.

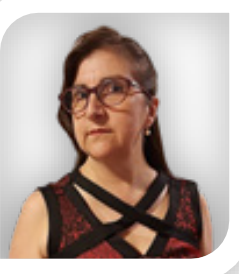

Doctora en Psicología Clínica graduada en la Universidad Central del Ecuador (UCE), obteniendo la maestría en Psicología con mención en Psicoterapia en la Universidad Internacional SEK (UISEK); realicé varios cursos de formación profesional en Psicología Forense a nivel Nacional e Internacional; Profesional Psicóloga con 20 años de experiencia como Psicoterapeuta en el área privada; en la actualidad me desempeño como Perito Psicóloga en el Consejo de la Judicatura en la Unidad de Violencia de la Mujer y la Familia, habiendo ejercido también las funciones de Perito Psicóloga en la Unidad de Peritaje Integral de la Fiscalía General del Estado; adicionalmente me desempeñé como Psicóloga del Centro de Rehabilitación Juvenil Virgilio Guerrero de la ciudad de Quito, Ecuador. 